\title{
Implementation of Novel Medical Image Compression Using Artificial Intelligence
}

\author{
Mohammad Al-Rababah \\ Department of computer sciences \\ Community College, Northern Border University, Saudi \\ Arabia
}

\author{
Abdusamad Al-Marghirani \\ Department of computer sciences \\ Faculty of Computing and Information Technology, \\ Northern Border University, Saudi Arabia
}

\begin{abstract}
The medical image processing process is one of the most important areas of research in medical applications in digitized medical information. A medical images have a large sizes. Since the coming of digital medical information, the important challenge is to care for the conduction and requirements of huge data, including medical images. Compression is considered as one of the necessary algorithm to explain this problem. A large amount of medical images must be compressed using lossless compression. This paper proposes a new medical image compression algorithm founded on lifting wavelet transform CDF 9/7 joined with SPIHT coding algorithm, this algorithm applied the lifting composition to confirm the benefit of the wavelet transform. To develop the proposed algorithm, the outcomes compared with other compression algorithm like JPEG codec. Experimental results proves that the anticipated algorithm is superior to another algorithm in both lossy and lossless compression for all medical images tested. The Wavelet-SPIHT algorithm provides PSNR very important values for MRI images.
\end{abstract}

Keywords-Medical image; lossless Compression; lifting wavelets; CDF9/7; Lifting scheme; SPIHT coding

\section{INTRODUCTION}

The current trend is the increasing use of digitized medical images [1,2] . Most of modern techniques of medical imaging produce 3D data ( MRI, CT , ultrasound, positron emission tomography ) and even 4D ( functional MRI, 3D echocardiography dynamic). Some images are intrinsically volume while others correspond on the contrary, a succession of images 2D (also called image stack ), to which is added a extra dimension, namely the difference between two successive cuts. In fact, the majority of medical images produced nowadays can be shown as images in at least three dimensions[3\}.

Medical images are constantly develop by giving a representation increasingly precise parts of the human body . However more image is more accurately the amount of data generated is large[1]. Medical images such as functional MRI, dynamic and tomography image dynamic 3D echocardiography is increasingly used as they are considered among the most effective techniques in medical imaging, but they produce the most voluminous data, hence the need for their compression for storage and / or transport through networks of telecommunication[4]..

Today, the large sizes use of numerical schemes in medical imaging (MRI, X scanner, nuclear medicine, etc.) generates massive volumes of data. However, Medical imaging play an important role on the diagnosis of diseases and surgical planning. These mega data need efficient transmission and long-term storage and [2]. So it is necessary to use algorithms of compression images in order to reduce the amount of data to be stored and transmitted.

For this reason, this paper is decomposed into three parts: the first part will present a representation of the Lifting scheme, then the second part present the biorthgonal wavelet CDF $9 / 7$ and finally and finally this paper present a SPIHT algorithm for medical image coding. In order to evaluate medical image compression by our algorithm, The PSNR results obtained are compared with the existing techniques namely JPEG codec[6].

This paper is organized as follows: Sect. 2 provides an overview of related works for medical image compression. Section 3 describes the proposed algorithm for medical image compression. In Sect. 4 experimental results are furnished and are compared with other algorithms. Finally, conclusions are drawn in Sect. 6.

\section{RELATED WORK}

In the medical image compression community [2], many technique for compression used like Lossless JPEG (Joint Photographic Experts Group) and lossless Wavelet [7]. JPEG formats is adopted by the Digital Imaging and Communications in Medicine (DICOM) group in their widely file format, but in the last years the wavelet compression algorithm give more performances than JPEG codec[4. In spite of this, a many image compression researches examine the use of compression for applying to medical images.

Currently, the compression in a radiology department is always performed without loss when it exists. It is performed by standard as lossless JPEG syntactically provided in the medical image DICOM1 format standard. This type of compression with a exact reconstruction of the original image, ensuring data integrity remains the preferred practitioners for obvious reasons of diagnosis. However it provides poor performance in terms of bit rate. The compression ratio (TC) potential vary about 2 to 8 next the information content of the image and the method applied [2,3]. The origin of the preference of doctors for lossless versus lossy compression is, as we said, avoid medical errors associated with poor image reconstruction. Indeed, the main problem of lossy compression for the medical images is because details important could 
disappear (others might possibly appear). These details are usually difficult to discern structures because they cause small changes in contrast

The standards of this family are known as JPEG and JPEG2000 names. The technique used for encoding still images can be reduced to two main families: method transform and structural engineering ( seeking uniformity in the image as : textures, contours, histogram). The structural approach uses techniques manipulate the intensity value of the pixel in the image. The two large families may be used together to a coding system. In other words, there is no border between Transform approach and structural approach. compression ratio is called the ratio between the sizes of the raw image and the compressed image[2].

\section{Proposed AlgorithM FOR MEdicAl IMAGE COMPRESSION}

In this paper proposed image compression algorithm is simulated using Color transform system and lifting wavelet CDF 9/7 where the compression is done using wavelet decomposition. This is analyzed to get the horizontal, vertical, approximation and diagonal details. The result is a lossless compression image. Wavelets coefficients are coded by the SPIHT algorithm coding[8] .In SPIHT algorithm it requires few bits to capture the same amount of information.

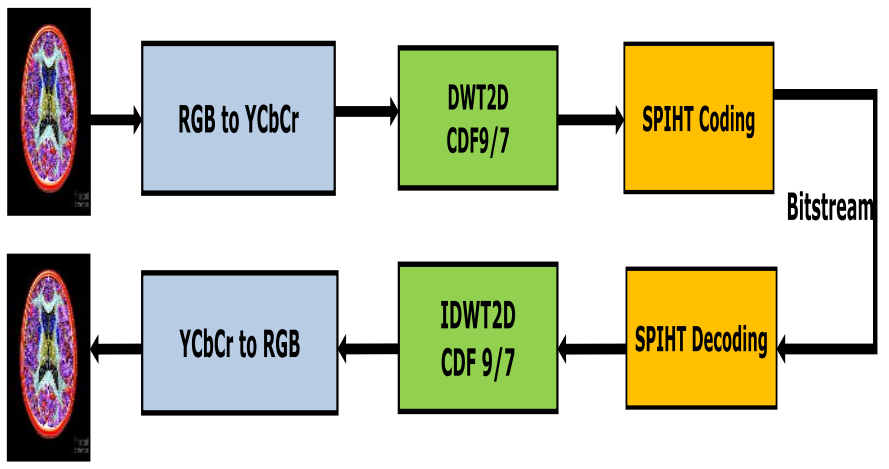

Fig. 1. Medical image Compression scheme

\section{A. Color Space Conversion}

According to the color space the image in a transformation of the components necessary to change color representation . The standard offers two modes to transform spend an RGB type representation (Red, Green, Blue) representation luminance $\mathrm{YCbCr}$ chrominance: transformed reversible or irreversible color. The irreversible transform ICT ( Irreversible Color Transform) is used in the case of a lossy compression . RCT reversible color transform ( Reversible Color Transform ) used in the case of lossless compression or lossy, is a approximation of the previous transformed [ 6 ]. The RGB to $\mathrm{YCbCr}$ is performed respecting to eq (1).

\footnotetext{
$\mathrm{Y}=0.257 \mathrm{R}+0.504 \mathrm{G}+0.098 \mathrm{~B}+16$

$\mathrm{Cb}=-0.148 \mathrm{R}-0.291 \mathrm{G}+0.439 \mathrm{~B}+128$

$\mathrm{Cr}=0.439 \mathrm{R}-0.368 \mathrm{G}-0.071 \mathrm{~B}+128$
} eq (2).

$$
\begin{aligned}
& \mathrm{R}=1.164(\mathrm{Y}-16)+1.596(\mathrm{Cr}-128) \\
& \mathrm{G}=1.164(\mathrm{Y}-16)-0.813(\mathrm{Cr}-128)-0.391(\mathrm{Cb}-128) \\
& \mathrm{B}=1.164(\mathrm{Y}-16)+2.018(\mathrm{Cb}-128)
\end{aligned}
$$

\section{B. Discrete Wavelet Transforms}

The discrete wavelet transform ( DWT ) is commonly used in the case of images 2D by application of separable filters in both directions [ 12 ]. The transformation Two-dimensional discrete wavelet ( 2 -D DWT ) is performed following separately the line order, then the order of the columns. We obtain four sub-bands by level of resolution : an approximation sub- band called LL and three sub-bands details LH, HH and $\mathrm{HL}$ respectively representing the horizontal details, and diagonal vertical image. Once the first level of decomposition performed, the transformed may be applied iteratively at each approximation LL sub band obtained for each level of resolution.

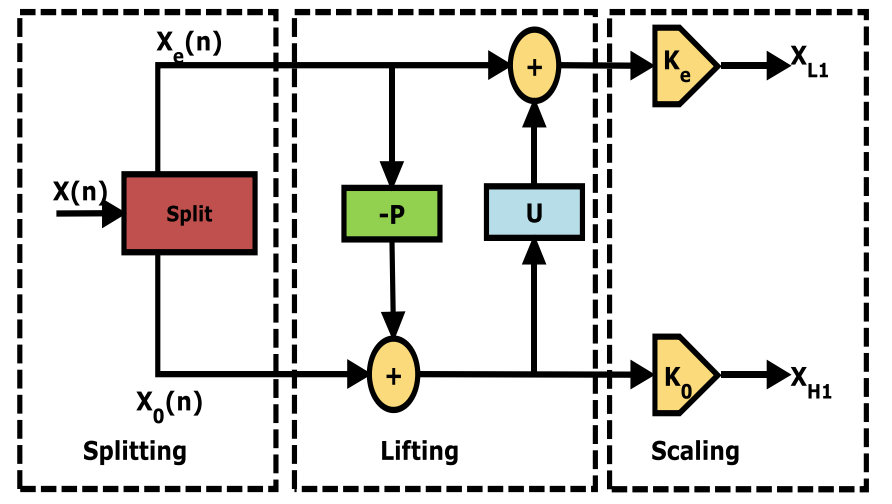

Fig. 2. The Lifting -based wavelet

1) Splitting: In this state, the original image $X$ is decomposed into two different parts, $X e(n)=X(2 n)$ and $X_{0}(n)$ $=X(2 n+1)$ that present respectively all even-indexed and oddindexed pixels of image $\mathrm{X}$ [6].

2) Lifting: In this state, using the prediction operation $P$, $\mathrm{X} 0(\mathrm{n})$ esteemed from $\mathrm{Xe}(\mathrm{n})$ and leads to an wrong signal $\mathrm{d}(\mathrm{n})$ that is the exact part of the original signal. Next, To update $\mathrm{d}(\mathrm{n})$ we applyed it to the update operation $\mathrm{U}$, and the obtained signal is united with $\mathrm{Xe}(\mathrm{n})$ to $\mathrm{s}(\mathrm{n})$ estimate, that represents the sleek part of the original signal [5]

3) Scaling: In this component, a normalization parameter is functional to $d(n)$ and $s(n)$, correspondingly. In the similarindexed part $\mathrm{s}(\mathrm{n})$ is improved by a normalization parameter Ke to produce the wavelet subband XL1 . In addition, in the odd-indicator part the fault signal $d(n)$ is enhance by $\mathrm{K} 0$ to obtain the wavelet subband XH1[14].

\section{Biorthogonal Wavelets CDF 9/7}

Biorthogonal wavelets $9 / 7$ part of the family symmetrical biorthogonal wavelets $\mathrm{CDF}$. The low-pass filters associated with $9 / 7$ wavelet thus have $p=9$ coefficients for the analysis, $p$ $=7$ coefficients to the synthesis and are described in Table I. The 9/7 biorthogonal wavelets are shown in Table 1 . 
TABLE I. A: THE ANALYSIS FILTER COEFFICIENTS

\begin{tabular}{|l|l|l|}
\hline \multicolumn{3}{|l|}{ Analysis filter coefficients } \\
\hline & Low-pass filter & High-pass filter \\
\hline 0 & 0.6029490182363579 & +1.115087052457000 \\
\hline \pm 1 & 0.6029490182363579 & +0.591271763114250 \\
\hline \pm 2 & +0.266864118442875 & -0.057543526228500 \\
\hline \pm 3 & -0.078223266528990 & -0.091271753114250 \\
\hline \pm 4 & -0.016864118442875 & \\
\hline
\end{tabular}

TABLE I.

B : THE SyNTHESIS FILTER COEFFICIENTS

\begin{tabular}{|l|l|l|}
\hline \multicolumn{3}{|l|}{ Synthesis filter coefficients } \\
\hline & Low-pass filter & High-pass filter \\
\hline 0 & +1.115087052457000 & 0.6029490182363579 \\
\hline \pm 1 & -0.591271763114250 & -0.266864118442875 \\
\hline \pm 2 & -0.057543526228500 & -0.078223266528990 \\
\hline \pm 3 & +0.091271763114250 & +0.016864118442875 \\
\hline \pm 4 & & +0.026748757410810 \\
\hline
\end{tabular}

Four states are used by the Lifting scheme of the biorthogonal transform 9/7. Two prediction operators and two update operators as presented in Fig.3. [13, 14].

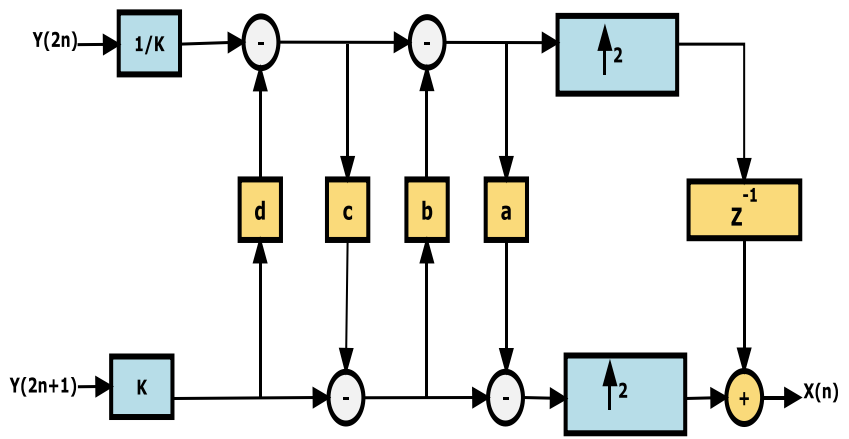

Fig. 3. Lifting implementation of the analysis side of the CDF $9 / 7$ wavelet

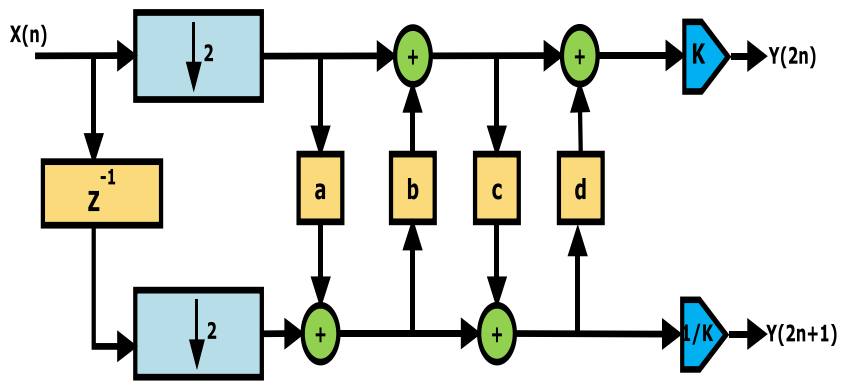

Fig. 4. Lifting implementation of the synthesis side of the CDF $9 / 7$ filter bank

\section{SPIHT Coding Scheme}

When wavelets coefficients for the decomposition image are obtained, the goal is to find a good algorithm to code the wavelet coefficients into an efficient result. The algorithm adopted in this paper is SPIHT algorithm[7]. The coder SPIHT (Set Partitioning In Hierarchical to Trees ) is an efficient algorithm for coding with and without loss the image.The SPIHT algorithm is composed by three steps:initialization, sorting pass and refinement pass. And it contain three lists: LIS list of insignificant sets ( LIS), list of insignificant pixels( LIP) and list of significant pixels (LSP). All nodes of the lowest frequency sub band are initialized in LIP stage[14]. In LIP each pixel is compared with the current threshold and a bit ( 0 or 1$)$ is generated to indicate wich pixel it is significant or not[11]. If a pixel in the offspring set is significant then it is moved to LSP and if it is insignificant moved to LIP and finally the bitsream of bits is generated[7].

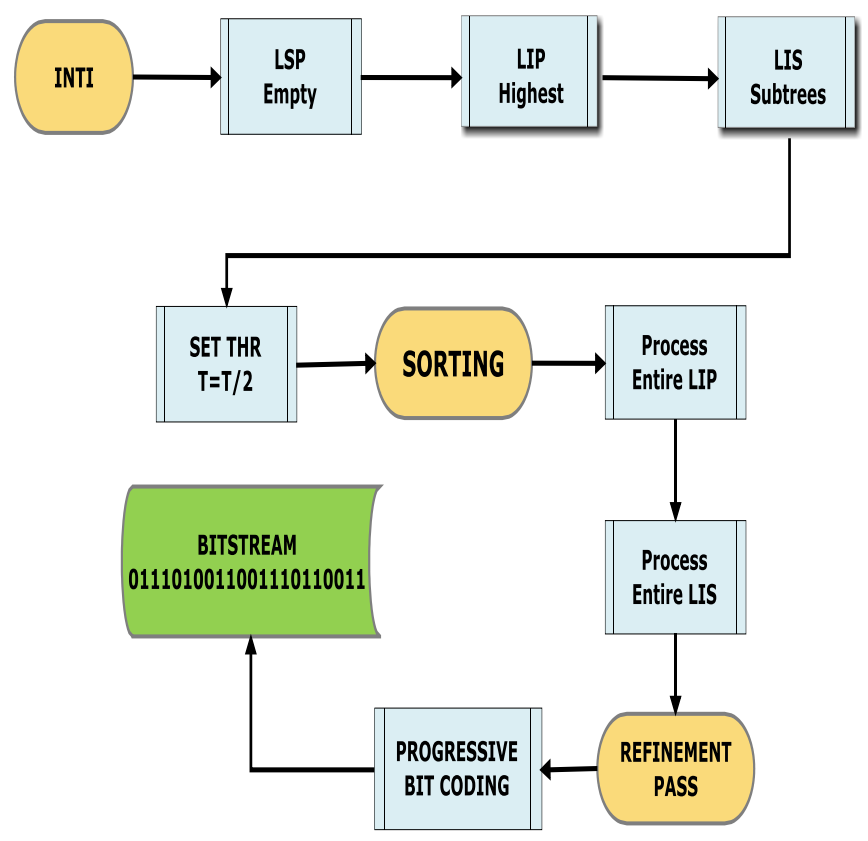

Fig. 5. Flowchart of SPIHT Algorithm

The SPIHT algorithm [2] is based on the principles whileproposing to partition recursively treescoefficients ( Figure 5). SPIHT performs partitioningrecursive of the shaft so as to determine the position ofsignificant coefficients in the offspring of the coefficientconsidered. Their sign is sent as soon as they are identifiedas signifiers and they are added to the list ofcoefficients to refine. This algorithm also worksby bit planes . It offers outstanding performance ,EZW without reaching those of entropy coding . By addingan entropy coding significance information, aadditional gain between 0.3 and 0.6 $\mathrm{dB}$ is obtained. Bitssent when the significance of the match passesprogram executed in the encoder during the execution ofthe ranking algorithm and significant coefficientsinsignificant.

\section{RESULTS AND DISCUSSION}

\section{A. Medical Image standard Test}

In this paper for lossless compression methods based on 2D lifting wavelet transforms and SPIHT coding. The simulation is doing on medical image, the spatial location and frequency are important [3, 4]. We applied our proposed algorithm on the tests color medicals images encoded by 24 bits per pixels(bpp). 

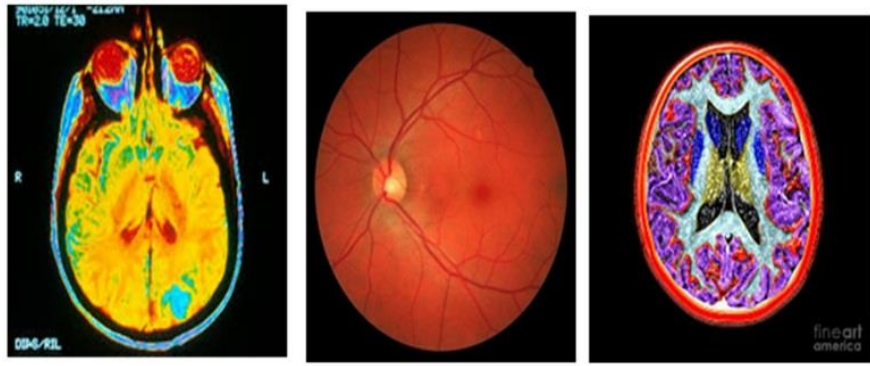

Fig. 6. Originals colors images

The goal of this simulationis to reducing the rates for which the medicals images quality remains acceptable. compressed image quality performances and judgments are given by the PSNR evaluation parameters.

\section{B. Compression Quality Evaluation}

PSNR is the most commonly used measure for performance testing methods compression of images with loss. It evaluates the image degradation rebuilt after compression by measuring the error between the original image and the image $\mathrm{X}$ rebuilt $\mathrm{Y}$ [1 ]. The unity of PSNR is the decibel $\mathrm{dB}$, the higher it is and the less distortion. PSNR is given by:

$$
P S N R=10 \log 10\left(\frac{(\text { Dynamic of } \operatorname{Im} \text { ages })^{2}}{M S E}\right)(3)
$$

For two $\mathrm{M} \times \mathrm{N}$ grayscale images $\mathrm{F}$ and $\hat{F}$, Mean Square Error (MSE) which requires, where one of the images is considered as a compression of the other is defined as:

$$
M S E=\frac{1}{M \times N} \sum_{i=1}^{i=M} \sum_{j=1}^{j=N}(F(i, j)-\hat{F}(i, j))^{2}(4)
$$

Results

The proposed codec is implemented using MATLAB 2013A for differents color images. This algorithm is simulated on different tests medical images. This image is taken from the GE Medical System database.

This algorithm is implemented with three level of decomposition for Discrete Wavelet transform(DWT). The results of three such images are shown here. Figure 6 present some results for simulation of different ratio (RC) values. According to the quality parameter PSNR,decoded imagegive a good PSNRfrom 0. 5bpp, As a result improvement in performance is observed at lower bit rates.

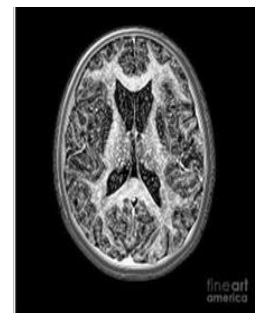
Bitrate $=0.05 \mathrm{bppp}$ Wavelet decomposition $=9$
compressed file $=$ d85160 bits
MSE $=2$ SNR $=34.7 \mathrm{~dB}$ PSNR $=54.1 \mathrm{~dB}$

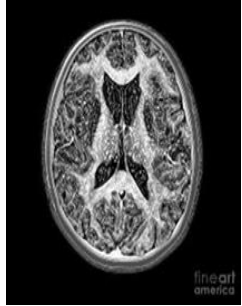

Bitrate $=0.5$ bpp Wavelet decomposition $=3$
compressed file 485161616 bits

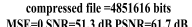

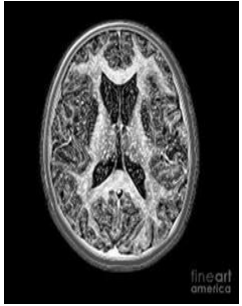

Bitrate $=0.5$ bpp Wavelet decomposition $=3$ compressed
file $=4851616$ bits YSE $=0$ SNR $=513$ 13 PSNR $=61.7 \mathrm{~dB}$

Fig. 7. PSNR results for medical images test

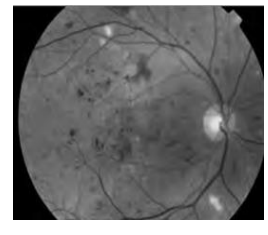

compressed file =221112 bits
MSE $=45 \mathrm{SNR}=15.2 \mathrm{~dB}$ PSNR $=31.6 \mathrm{~dB}$

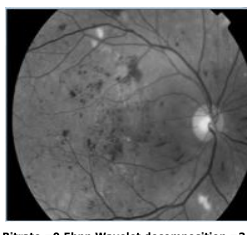

Bitrate =0.5bpp Wavelet decomposition $=3$
MSE compressed file =2211112
MSts $=12.1$ SNR $=20.9 \mathrm{~dB}$ PSNR $=37.3 \mathrm{~dB}$

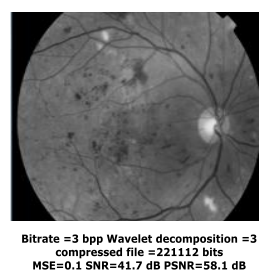

Fig. 8. PSNR results for medical images test

To show the performance of the proposed method, we make a comparison between these different types of transform CDF9/7 (lifting scheme)) coupled with SPIHT coding and JPEG coder, which we calculate the various parameters (PSNR) in order to study the influence of coding choices on color medical images. For each application we vary the bit-rate from 0.05 to 2 , and we calculate the PSNR. The results obtained are given in Table II.

TABLE II. COMPARISON WITH EXISTING METHOD (JPEG CODER)

\begin{tabular}{|l|l|l|l|l|l|}
\hline JPEG & \multicolumn{3}{|c|}{ Proposed Algorithm } \\
\hline bpp & CR & PSNR(dB) & bpp & CR & PSNR(dB) \\
\hline 0.05 & 160 & 23.5 & 0.05 & 160 & 33 \\
\hline 0.3 & 26 & 32.3 & 0.3 & 26 & 34.4 \\
\hline 0.75 & 10 & 34.6 & 0.75 & 10 & 42.5 \\
\hline 1 & 8 & 41.3 & 1 & 8 & 44.7 \\
\hline 2 & 4 & 44.5 & 2 & 4 & 47.9 \\
\hline
\end{tabular}

\section{CONCLUSION}

This paper present a fresh algorithm for medical image compression. This algorithm is based on lifting wavelet transforms and SPIHT code. The algorithm provides awfully essential PSNR values for MRI images and it is more appropriate for this type of images. Accordingly, we bring to a close that the results obtained by our compression codec are incredibly agreeable in terms of compressed image quality (PSNR) and compression ratio . In perception, we desire to revise our algorithm for applaying to compress medical image 3D and 4D. 


\section{REFERENCES}

[1] T. H. Oh, H. S. Lim, and S. Y. Pang, "Medical image processing: from lossless to lossy compression," in Proceedings of the IEEE Region 10 Conference (TENCON '05), pp. 1-6, November 2005.

[2] M kaur and Wasson V , Region of Interest based Compression techniques for Telemedicine Application, International Journal of Research in Electronics and Computer Engineering, 2015:Vol. 3, pp. 63-67.

[3] BairagiVinayakK., and Ashok Sapkal M. ROI-based DICOM image compression for telemedicine. Sadhana, 2013; Vol. No.38, pp.123- 131.

[4] Devi S. S. \&VidhyaK.. Development of medical image compression techniques. In IEEEConference on Computational Intelligence and Multimedia Applications, 2007; Vol. 3, pp. 97-101.

[5] Daubechies, W. Sweldens: Factoring Wavelet Transforms into Lifting Steps, Journal of Fourier Analysis and Applications, Vol.4, No. 3, May 1998 , pp. $247-269$.

[6] P. Wu P., Xie K., Yu H., Zheng Y. and Mao Yu W.A New Preprocessing Algorithm Used in Color Image Compression..Advances in Future Computer and Control Systems. Springer Berlin Heidelberg, 2012;pp.465-471.

[7] S.G. Miaou, S.T. Chen, S.N. Chao: Wavelet-based Lossy-to-lossless Medical Image Compression using Dynamic VQ and SPIHT Coding, Biomedical Engineering: Applications, Basis \& Communications, Vol. 15, No. 6, Dec. 2003, pp. $235-242$.
[8] D.S. Taubman, M.W. Marcellin: JPEG2000: Image Compression Fundamentals, Standards and Practice, Kluwer Academic Publishers, London, 2002.

[9] G. Pau: Advanced Wavelets and Space-time Decompositions: Application to Video Coding Scalable, Phd thesis, National School of Telecommunications, Paris, 2006.

[10] Savakis, R. Carbone: Discrete Wavelet Transform Core for Image Processing Applications, Real-time Imaging IX, SPIE-IS\&T Electronic Imaging, San Jose, CA, USA, SPIE Vol. 5671, Jan. 2005, pp. 142 - 151.

[11] A.M. Lakhdar, R. Méliani, M. Kandouci: Robust Image Transmission Performed by SPIHT and Turbo-codes, Serbian Journal of Electrical Engineering Vol. 5, No. 2, Nov. 2008, pp. 353 - 360.

[12] Sure. Srikanth, Sukadev Meher, "Compression Efficiency for Combining Different Embedded Image Compression Techniques with Huffman Encoding", International conference on Communication and Signal Processing, April 3-5, 2013, India,IEEE.

[13] Salija.P , Manimekalai.M.A.P, N.A Vasanthi, PhD. "ROI and SeamSPIHT based Efficient Image Compression for Mobile Multimedia and Medical Applications", International Journal of Computer Applications (0975 - 8887) Volume 64- No.12, February 2013.

[14] K. Kannan, S.A. Perumal, K. Arulmozhi: Optimal Decomposition Level of Discrete, Stationary and Dual Tree Complex Wavelet Transform for Pixel based Fusion of Multi-focused Images, Serbian Journal of Electrical Engineering Vol. 7, No. 1, May 2010, pp. 81 - 93. 